\title{
SCIDOC
}

International Journal of Dentistry and Oral Science (IJDOS)

ISSN: 2377-8075

\section{Awareness Of Psoriasis Among General Population - A Questionnaire Based Study}

Research Article

Dhanraj Ganapathy ${ }^{1 *}$, Harshinee Chandrasekhar ${ }^{2}$, Brundha ${ }^{3}$

${ }^{1}$ Professor \& Head of Department Department of Prosthodontics, Saveetha Dental College and Hospitals Saveetha Institute of Medical and Technical Sciences Saveetha University, Chennai, India.

${ }^{2}$ Undergraduate student Saveetha Institute of Medical and Technical Sciences Saveetha University, Chennai, India.

${ }^{3}$ Associate Professor Department of General Pathology Saveetha Institute of Medical and Technical Sciences Saveetha University, Chennai, India.

\section{Abstract}

Background: Psoriasis is an autoimmune disease that affects the skin and mucous membranes and the Oral manifestations of psoriasis are not well recognized like skin lesions. As Psoriasis may be a rare oral manifestation but it is very important to know about it.

Aim: To carry on a survey to know about the knowledge of Psoriasis among the general population.

Materials And Methods: 100 students were chosen and a questionnaire including 6 questions were surveyed and the results are obtained. The questionnaire included questions concentrating on the knowledge of the students about the knowledge of Psoriasis.

Results and Conclusion: The awareness of Psoriasis disease among the general population was taken as a study and it was found that upto $90 \%$ have a knowledge of this disease. But the knowledge on the diagnosis, treatment and prevention was minimum which has to be improved.

Keywords: Awareness; Population; Skin; Oral; Psoriasis.

\section{Introduction}

Psoriasis is an autoimmune disease that affects the skin and mucous membranes and the Oral manifestations of psoriasis are not well recognized like skin lesions.

In patients with psoriasis, a cell's life cycle is three to six days; mature cells move rapidly to the skin surface and are non-vital. This layering of cells presents clinically as a whitish, flaky crust [6].

Oral psoriasis has been seen to manifest in broadly four types of lesions:

(1) well defined yellowish $\square$ white lesions, round to oval in shape, which are independent of cutaneous psoriasis.
(2) white, lacy, circinate, elevated lesions on the mucosa and tongue that are congruent with skin lesions.

(3) erythema or redness of the entire oral mucosa associated with acute exacerbation of psoriasis.

(4) geographic tongue, seen more frequently in patients with cutaneous psoriasis than in controls [5].

In recent studies, they have found some etiological factors which can cause psoriasis, they are:

Infections such as streptococcal infections and/or tonsillitis.

Patients diagnosed with HIV may exhibit a more severe level of psoriasis.

\section{*Corresponding Author}

Dhanraj Ganapathy,

Professor \& Head of Department Department of Prosthodontics, Saveetha Dental College and Hospitals Saveetha Institute of Medical and Technical Sciences Saveetha University, 162,P. H. Road, Chennai-600077 TamilNadu, India. Tel: +919841504523

Received: February 25, 2021

Accepted: March 04, 2021

Published: March 18, 202

Citation: Dhanraj Ganapathy, Harshinee Chandrasekhar, Brundha. Awareness Of Psoriasis Among General Population - A Questionnaire Based Study. Int J Dentistry Oral Sci. 2021;08(03):2108-2111. doi: http://dx.doi.org/10.19070/2377-8075-21000416

Copyright: Dhanraj Ganapathy ${ }^{\circ} 2021$. This is an open-access article distributed under the terms of the Creative Commons Attribution License, which permits unrestricted use, distribution and reproduction in any medium, provided the original author and source are credited. 
Cold and dry weather increase chances of outbreak, while hot, sunny weather appears to help control symptoms.

Trauma to skin, including cuts, bruises, burns, vaccinations, tattoos, may cause flare-up either at the site of trauma or elsewhere on the body.

Drugs prescribed for malaria, some beta-blockers and lithium, common treatment for bipolar disorder, can cause flare-up.

NSAIDs may also aggravate psoriasis.

Alcohol use may increase chances of flare-up in men.

Tobacco use may worsen psoriasis [2].

Psoriasis can be initially treated with topical corticosteroids, coal tar, keratolytic agents, vitamin D-3 analogs and topical retinoids in an attempt to reduce the inflammatory response and relieve symptoms of itching and redness. A review of these drugs did not reveal any dental side effects. But some drugs like methotrexate and cyclosporine have the effects on oral cavity like ulcerative stomatitis, gingivitis, glossitis, mouth sores, swallowing difficulty, gum hyperplasia, xerostomia, abnormal taste, tongue disorder and gingival bleeding [3].

\section{Materials and Method}

This questionnaire based study was conducted for a period of one month (December 2016). Questions about the knowledge of psoriasis were asked to 100 people of the general population and the cause, symptoms and the treatment of psoriasis. The questionnaire consists of 6 questions about the knowledge of psoriasis. The purpose of this study is to spread awareness among the general population about the knowledge of psoriasis.

The questions in the questioner are as follows,

1. Have you heard of Psoriasis? Yes/No

Sources:- Friend.

Family.

Graph 1. The awareness of Psoriasis among general population was taken and it was found that $90 \%$ of the population have a knowledge of this disease.

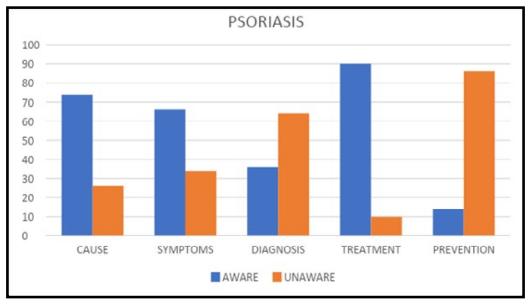

Graph $2.74 \%$ of the respondents reacted positively to the causes of Psoriasis and the medications (20\%) was the major cause according to them.

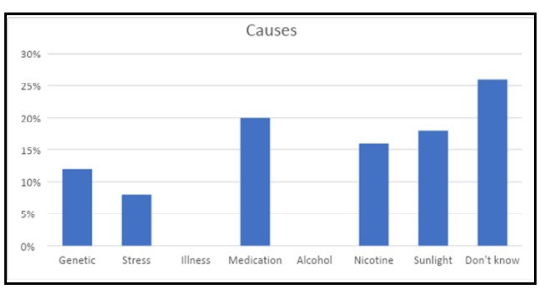

Graph 3. The questionnaire consisted symptom options such as red patches, ulcers, peeling gums and redness of mucous membrane. It is surveyed that $66 \%$ of the population have a knowledge of some of these symptoms with a high positive response given to the ulcer option.

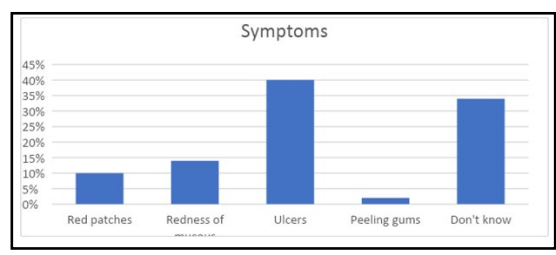

Graph 4. 72\% of the respondents know the diagnostic method of Psoriasis and the response was biopsy.

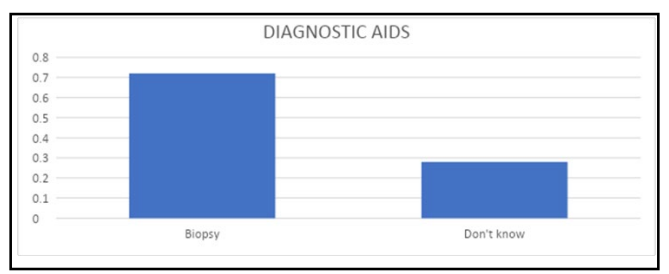


Media.

2. Do you know about the causes of Psoriasis? Yes/No Causes:- Genetic.

stress.

illness.

medication.

alcohol.

nicotine.

sunlight.

3. Do you know the symptoms of Psoriasis? Yes/No

If yes, what are the symptoms:-

Red patches with red or white borders.

Redness of the mucus membranes in the mouth.

Pustules or ulcers.

Peeling gums.

None of the above.

Don't know.

4. Do you know the diagnostic methods for Psoriasis? Yes/No

If yes, what are the methods:-

Biopsy.

Don't know.

5. Do you know the treatment for Psoriasis? Yes/No

If yes, what are treatment :-

Topical cortical steroids.

Antiseptic mouthwash.

Medicines.

Don't know.

6. Do you know the way to prevent Psoriasis? Yes/No

If yes, which of the following methods will be useful:-

Maintain good health.

Avoid alcohol.

Meditation.

Don't know.

\section{Results}

Graph 1 to Graph 6.

\section{Discussion}

Psoriasis is related to immune system problems with T cells and other white blood cells. Psoriasis can be triggered by factors like Stress, smoking, sunlight and infections. Psoriasis can also be caused genetically. Psoriasis can also be induced by drugs, some of the drugs that are responsible for psoriasis are Acetazolamide, Atenolol, Chloroquine, Cyclosporin, Diltiazem, Lithium, propranolol and Terbinafine. Roujeau et al. report that within a small population of 63 patients, medications could be responsible for psoriasis in as many as $83 \%$ of cases [10]. According to Braun Falco et al., some of the most common medications known to trigger or worsen existing psoriasis include lithium, gold salts, beta blockers and antimalarials [4].

Psoriasis occurs in skin and the oral mucosa, skin is a common area of occurrence and it rarely occurs on mucosa. The characteristic features of psoriasis on skin appears as patches of raised, reddish skin covered by a silvery-white scale. These patches frequently form on the elbows, knees, lower back and scalp [9].

The intraoral characteristics of psoriasis appear on the lips, tongue, palate, buccal mucosa and gingiva. Oral manifestations of oral psoriasis include small, whitish papules with bleeding points when scraped; red and white plaques that follow skin lesions; and bright red patches. White lesions on the oral mucosa may change in severity daily. Psoriasis can also be seen in conjunction with angular cheilitis when they appear at the commissures and perioral tissues. Geographic tongue lesions and fissured tongue are seen in patients with psoriasis. Geographic tongue which is also known as benign migratory glossitis, is a creeping area of diffused redness surrounded by a white, hyperkeratotic border with atrophy of the filiform papillae. In general, oral manifestations are asymptomatic and may not be reported. It is also possible that the oral environment itself may alter oral lesions, both clinically and histologically.

Graph 5. 90\% of the population have knowledge on the treatment of Psoriasis with a high response given to medicines $(82 \%)$.

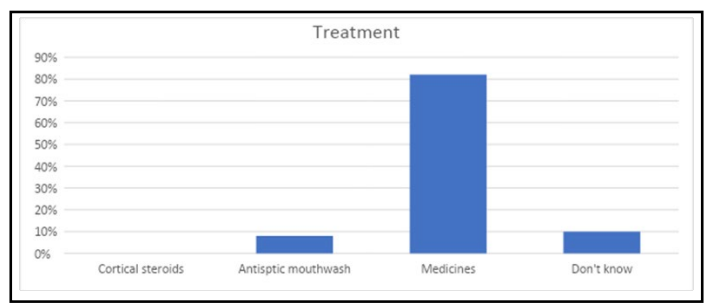

Graph 6. There is a less knowledge about the prevention of psoriasis which is by maintaining a good health (14\%).

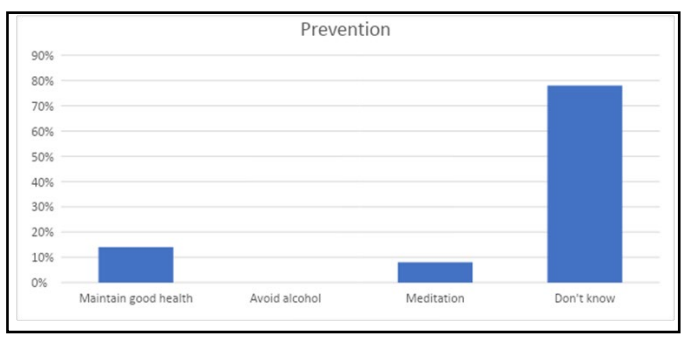


Patients with arthritic psoriasis, squeezing a tube of toothpaste may be painful and gripping a toothbrush handle may be difficult [8].

Diagnosis of psoriasis can be done by taking proper medical history and clinical examination of skin, scalp and nails. Biopsy can be used for diagnosis of psoriasis, skin biopsy is used as a diagnostic method for psoriasis.

Treatment for psoriasis can be given by topical ointments, light therapy and medications. Medications which are used for treating psoriasis are steroids, anti inflammatory drugs, vitamin supplements. Antiseptic mouthwash is used in oral psoriasis [11].

Topical corticosteroids are still used for topical psoriasis treatment despite the development of newer agents. Corticosteroids exert anti inflammatory, antiproliferative, and immunosuppressive actions by affecting gene transcription. Topical corticosteroids generally can be continued as long as the patient has thick active lesions. Skin atrophy from topical corticosteroids usually is not a problem unless the medication is continuously applied after the skin has returned to normal thickness. Once clinical improvement occurs, the frequency of application should be reduced [7].

Topical vitamin D analogs for the treatment of psoriasis include calcipotriene, calcitriol, and tacalcitol. Although topical vitamin D analogs are effective as monotherapy for some patients, a systematic review found that combination therapy with a topical corticosteroid is more effective than either treatment alone [1].

\section{Results and Conclusion}

The awareness of Psoriasis disease among the general population was taken as a study and it was found that upto $90 \%$ have a knowledge of this disease. But the knowledge on the diagnosis, treatment and prevention was minimum which has to be improved.

\section{References}

[1]. Ashcroft DM, Po AL, Williams HC, Griffiths CE. Systematic review of comparative efficacy and tolerability of calcipotriol in treating chronic plaque psoriasis. BMJ. 2000 Apr 8;320(7240):963-7. Pubmed PMID: 10753146.

[2]. Brice DM, Danesh-Meyer MJ. Oral lesions in patients with psoriasis: clinical presentation and management. J Periodontol. 2000 Dec;71(12):1896-903. Pubmed PMID: 11156048.

[3]. Bruce AJ, Rogers RS 3rd. Oral psoriasis. Dermatol Clin. 2003 Jan;21(1):99104. Pubmed PMID: 12622272.

[4]. Christophers, Enno, and Ulrich Mrowietz."Psoriasis." Braun-Falco's Dermatology. 2009. https://doi.org/10.1007/978-3-540-29316-3_36.

[5]. Kalb RE, Bagel J, Korman NJ, Lebwohl MG, Young M, Horn EJ, Van Voorhees AS; National Psoriasis Foundation. Treatment of intertriginous psoriasis: from the Medical Board of the National Psoriasis Foundation. J Am Acad Dermatol. 2009 Jan;60(1):120-4. Pubmed PMID: 19103363.

[6]. Lui H, Mamelak AJ. Plaque psoriasis. Medscape reference. 2018.

[7]. Mason AR, Mason J, Cork M, Dooley G, Hancock H. Topical treatments for chronic plaque psoriasis. Cochrane Database of Systematic Reviews. 2013(3).

[8]. Menter, A., N. J. Korman, C. A. Elmets, S. R. Feldman, J. M. Gelfand, K. B. Gordon, A. Gottlieb, et al. 2009. "American Academy of Dermatology. Guidelines of Care for the Management of Psoriasis and Psoriatic Arthritis. Section 3. Guidelines of Care for the Management and Treatment of Psoriasis with Topical Therapies." Journal of the American Academy of Dermatology 60 (4): 643-59.

[9]. Rongioletti F, Fiorucci C, Parodi A. Psoriasis induced or aggravated by drugs. The Journal of Rheumatology Supplement. 2009 Aug 1;83:59-61.

[10]. Roujeau JC, Bioulac-Sage P, Bourseau C, Guillaume JC, Bernard P, Lok C, Plantin P, Claudy A, Delavierre C, Vaillant L, Wechsler J. Acute generalized exanthematous pustulosis: analysis of 63 cases. Archives of Dermatology. 1991 Sep 1;127(9):1333-8.

[11]. Samarasekera EJ, Sawyer L, Wonderling D, Tucker R, Smith CH. Topical therapies for the treatment of plaque psoriasis: systematic review and network meta-analyses. British Journal of Dermatology. 2013 May;168(5):95467. 